\title{
Statistical Model of OFDMA Cellular Networks Uplink Interference Using Lognormal Distribution
}

\author{
Jianhua He, Senior Member, IEEE, Zuoyin Tang, Hsiao-Hwa Chen, Fellow, IEEE, and Wenqing Cheng
}

\begin{abstract}
In this letter, we propose an analytical approach to model uplink intercell interference (ICI) in hexagonal grid based orthogonal frequency division multiple access (OFMDA) cellular networks. The key idea is that the uplink ICI from individual cells is approximated with a lognormal distribution with statistical parameters being determined analytically. Accordingly, the aggregated uplink ICI is approximated with another lognormal distribution and its statistical parameters can be determined from those of individual cells using FentonWilkson method. Analytic expressions of uplink ICI are derived with two traditional frequency reuse schemes, namely integer frequency reuse schemes with factor 1 (IFR-1) and factor 3 (IFR3). Uplink fractional power control and lognormal shadowing are modeled. System performances in terms of signal to interference plus noise ratio (SINR) and spectrum efficiency are also derived. The proposed model has been validated by simulations.
\end{abstract}

Index Terms-Frequency reuse scheme; cellular network; performance modeling; uplink interference; OFDMA

\section{INTRODUCTION}

In OFDMA systems, available spectrum resources are split up into a number of parallel orthogonal narrow-band subcarriers [1]. In an OFDMA based network, subcarriers are uniquely allocated to mobile users within a cell, which eliminates intracell interference but may not help to remove intercell interference (ICI). ICI has a great impact on the overall network performance as well as individual user performance. Various frequency reuse schemes have been proposed for OFDMA networks to mitigate ICI [2]. In frequency reuse schemes, an important design and planning issue is to balance the overall spectrum efficiency and cell-edge user data rate. Accurate and thorough analysis of the intercell interference is believed to be a key to address the issue.

In the literature, there have been extensive research works on modeling downlink ICI. Typical models include a simplified and yet effective scheme for its use in one-tier of interfering cells [3], and a fluid model for hexagonal grid based cellular networks (see [4] and its references), which assumed base stations continuously distributed in a network without considering cell boundaries. Recently, there is an increasing research effort in modeling downlink ICI using stochastic geometry model, e.g., [2] [5]. For uplink ICI modeling, the problem is much more challenging due to the fact that the positions of interfering mobiles are not fixed. A widely used model for the uplink ICI is Wyner model [6], in which intercell

Jianhua He (e-mail: j.he7@aston.ac.uk) and Zuoyin Tang (e-mail: $z . t a n g 1$ easton.ac.uk) are with the School of Engineering and Applied Science, Aston University, UK. Hsiao-Hwa Chen (the corresponding author) (e-mail: hshwchen@mail.ncku.edu.tw) is with the Department of Engineering Science, National Cheng Kung University, Taiwan. Wenqing Cheng (e-mail: chengwenqing@gmail.com) is with the Department of Electronics and Information Engineering, Huazhong University of Science and Technology, China. interference was assumed to be a weight of aggregate signals transmitted from adjacent cells. It was shown in [7] that the Wyner model is inaccurate for cellular networks employing TDMA or OFDMA technologies. Elayoubi et al. studied the uplink ICI and capacity in 3G LTE systems without shadowing, but the approach of calculating the aggregate interference and verification of the analytic model were not presented [8]. Karray studied the feasibility conditions for uplink resource allocation in both CDMA and OFDMA networks [9]. The uplink ICI was computed by approximation with an interference factor, similar to the idea used by Wyner for CDMA networks. The ICI analysis was not verified in [9]. The fluid model was used to analyze uplink ICI and uplink power compensation, but the model was not verified either [10]. A framework of modeling uplink ICI was reported in [11]. The model was very complex and requires special software packages such as Mathematica and MAPLE to get numerical results. It was proposed for single tier cells and did not consider uplink power compensation.

In this letter, we propose an approach to analyze the statistics of uplink intercell interference in OFDMA based hexagonal cellular networks. We approximate uplink interference from individual cells as a lognormal random variable. The aggregate uplink intercell interference from all interference cells is further approximated as another lognormal random variable. We derive analytic expressions of single cell ICI statistics for the IFR-1 and IFR-3 schemes with power compensation and shadowing modeling, and use Fenton-Wilkson method to determine the statistics parameters for aggregated interference. System performances in terms of SINR and spectrum efficiency are also derived. To the best of our knowledge, single cell uplink ICI has not been modeled using lognormal distribution before. Compared to the existing analytic models, our approach is simple and yet effective. It can be used for arbitrary number of tiers and irregular cell shapes. The model can provide a useful tool for evaluation and management of uplink ICI in OFDMA based networks.

\section{SySTEM MODEL}

\section{A. Frequency reuse schemes}

In this letter, we consider two frequency reuse schemes, namely IFR-1 and IFR-3, for the theoretic study of uplink ICI. The proposed analytic approach is generic and can be extended for the analysis of other frequency reuse schemes, such as FFR and SFR [2]. The IFR-1 and IFR-3 schemes have been widely used in multi-cell networks. The main objective of modern cellular networks is to achieve a high spectral efficiency with frequency reuse factor one. However, the IFR-1 scheme has major problems that intercell interference limits the capacity 
of the overall network and the users located in border areas suffer from a strong interference and low data rate. In the IFR-3 scheme, system bandwidth is divided equally into three sub-bands. Each cell is allocated one sub-band different from those allocated to its surrounding cells.

\section{B. Power control and channel model}

Similar to frequency reuse schemes, uplink power control has a great impact on achieving a required SINR, while at the same time controlling the interference caused to neighboring cells. In a classic power control scheme, all users are expected to receive the same SINR in uplink. An alternative power control scheme, called fractional power control (FPC), was approved by 3GPP, in which users with a higher path-loss can operate at a lower SINR and thus generate less intercell interference [12].

In this letter, the FPC scheme is used in the system. Consider the transmission from a user over a physical resource block (PRB), which represents a basic time-frequency resource unit available for data transmission. In the FPC scheme, under a simple signal propagation model, the signal to noise ratio (SNR) from a user located $r$ meters away from its serving BS can be written as

$$
S N R=\frac{P_{t} r^{\alpha(\beta-1)} \psi}{\delta^{2}},
$$

where $P_{t}$ denotes the baseline transmit power for a PRB irrespective of user locations, $\alpha$ is the path loss exponent, $\delta^{2}$ is noise power, and $\beta$ is the power control factor [12]. It is noted that there is no power compensation on the signal path loss with $\beta=0$. On the other hand, path loss is fully compensated with $\beta=1$. $\psi$ denotes log-normal shadowing fading between a user and its BS. In this article, we assume that the normal distributions associated with the log-normal shadowing for the uplinks between all the users and BSs have a zero mean and a common standard deviation $\sigma_{w}$.

\section{AnALYTIC MODEL}

Let us consider the uplink ICI generated on a single PRB to the users located in a target cell (say cell 0 ). Assume that users are uniformly distributed in hexagonal cells, although our model can be used for irregularly shaped cells as well. The users in each cell are supposed to be served randomly and independently by their serving BSs with an equal opportunity in terms of usages in both time and PRBs. We consider only the interference from users within the closest $N_{t}$ tiers of cells centered on the target cell $\left(N_{t} \geq 1\right)$, which have $N_{c}$ cells in total. The target BS is located at the origin. BSs are assumed to use omni-directional antennas. Cell radius is denoted by $R_{c}$ and the half distance between two neighbor BSs is denoted by $R_{b}$. A heavy loaded OFDMA cellular network is assumed, in which the PRBs allocated to each cell are fully utilized.

Let $I_{n}$ denote the uplink ICI generated from cell $n(1 \leq n \leq$ $N_{c}$ ) without consideration of shadowing, which is assumed to be a lognormal distributed random variable. The ICI $I_{n}$ can be uniquely characterized by its mean and variance, which are denoted by $\overline{I_{n}}$ and $\widehat{I_{n}}$, respectively. Let $\mu_{n}$ and $\sigma_{n}^{2}$ denote the mean and variance of the normal distribution associated with $I_{n}$, respectively, which can be calculated from $\overline{I_{n}}$ and $\widehat{I_{n}}$ by

$$
\begin{gathered}
\sigma_{n}^{2}=\ln \left(1+\frac{\widehat{I_{n}}}{\overline{I_{n}}}\right), \\
\mu_{n}=\ln \left(\overline{I_{n}}\right)-\frac{\sigma_{n}^{2}}{2} .
\end{gathered}
$$

Then, the probability density function of $I_{n}$ can be expressed by

$$
f_{I_{n}}\left(x ; \mu_{n}, \sigma_{n}\right)=\frac{1}{x \sigma_{n} \sqrt{2 \pi}} e^{-\frac{\left(\ln x-\mu_{n}\right)^{2}}{2 \sigma_{n}^{2}}} .
$$

Let $I_{w, n}$ denote the uplink ICI generated from cell $n$ ( $1 \leq n \leq N_{c}$ ) with shadowing. We have $I_{w, n}=I_{n} \psi_{n}$, where $I_{n}$ represents the single cell interference generated by exactly one user from cell $n$ without shadowing, and $\psi_{n}$ represents the lognormal shadowing between the user from cell $n$ and BS 0. It is easy to show that $I_{w, n}$ is a lognormal distributed random variable. Let $\mu_{w, n}$ and $\sigma_{w, n}$ denote the mean and standard deviation of the normal distribution associated with $I_{w, n}$. Then, we have $\mu_{w, n}=\mu_{n}$ and $\sigma_{w, n}^{2}=\sigma_{n}^{2}+\sigma_{w}^{2}$.

To characterize the statistics of the aggregated uplink ICI in an OFDMA network, we first derive the mean and variation of ICI from each individual interfering cell. Then, we derive the aggregated ICI from all interfering cells, which can be approximated by a lognormal random variable as well. Let $I_{\text {sum }}$ denote the aggregated ICI, which can be calculated by $I_{s u m}=\sum_{n=1}^{N_{c}} I_{w, n}$. Let $\bar{I}_{\text {sum }}$ and $\widehat{I}_{s u m}$ denote the mean and variation of $I_{\text {sum }}$, respectively, and $\mu_{\text {sum }}$ and $\sigma_{\text {sum }}$ denote the mean and standard deviation of the normal distributions associated with $I_{\text {sum }}$, respectively.

\section{A. Mean and standard deviation of uplink ICI}

We start with the derivation of the mean and standard deviation of the lognormal distributed single cell ICI for IFR-1 scheme. Let $N_{f r 1}(l)$ denote the number of interfering cells in the $l$ th tier of the IFR-1 scheme with $N_{\text {cell }}(l)=6 l$.

Without loss of generality, we derive the uplink ICI from cell $n$ (let $l$ be the tier ID of cell $n$ ) for $1 \leq n \leq N_{c}$. For simplicity, assume that in the analytic model, each BS in the $l$ th tier has an equivalent distance $D_{b}(l)$ to the target BS. $D_{b}(l)$ is approximated by $2 l R_{b}$ for an odd number $l$ and $1.5 l R_{c}$ for an even number $l$. Such approximation has a negligible impact on the model accuracy, as shown in the model verification results.

Consider a generic infinitely small element surface in an interfering cell, which has a distance $r$ to the BS of the interfering cell and an angle $\theta$ to the directed line connecting the target BS to the BS of interfering cell. The area of this element surface is supposed to be $r d r d \theta$. An illustration of the location of the element surface is shown in Fig. 2 for Cell 18 in the second tier. Let $R_{u}(n, r, \theta)$ denote the distance between the considered element surface and the target BS, which is computed by

$$
R_{u}(n, r, \theta)=\sqrt{D_{b}(l)^{2}+r^{2}-2 r D_{b}(l) \cos (\pi-\theta)} .
$$

Let $\rho_{f r 1}$ denote the user density in the IFR-1 scheme. We have $\rho_{f r 1}=\frac{1}{\pi R_{v}^{2}}$. Let $I(n, r, \theta)$ denote the contribution of 


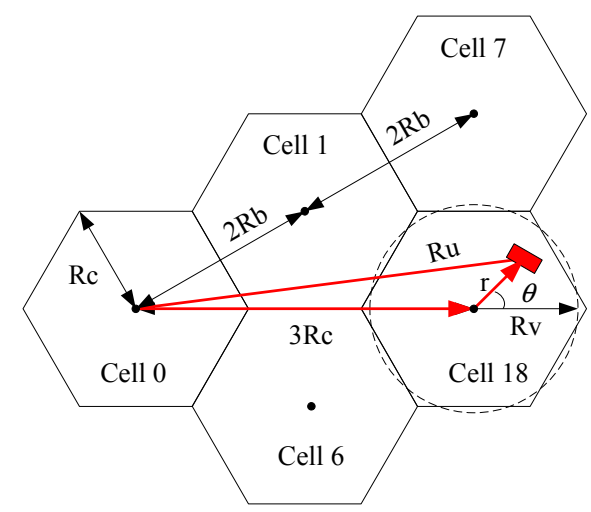

Fig. 1. An example calculation of uplink interference contributed from an element surface in cell 18 of the 2 nd tier.

uplink interference from this general element surface, which can be computed by

$$
I(n, r, \theta)=P_{t} r^{\beta \alpha} R_{u}(n, r, \theta)^{-\alpha} \rho_{f r 1} r d r d \theta,
$$

where $r^{\beta \alpha}$ represents the scale of compensated power. It is worth noting that in a real system for both simulation and analysis, we assumed that there is exactly one interfering user per cell at any time. Therefore, there is no intracell interference. The interference contribution from small size element surface is used solely to calculate the mean and standard deviation of lognormal distributed single cell interference.

To calculate the mean of the ICI from the whole cell $n$, we use a disk cell with its radius $R_{v}$ for integration instead of a hexagon cell. $R_{v}$ is approximated by $\left(R_{c}+R_{b}\right) / 2$. It is known that such approximation has a negligible impact on the model accuracy. With this approximation, we can calculate the mean uplink ICI $\overline{I_{n}}$ by integrating $I(n, r, \theta)$ over a disk of size $R_{v}$, or

$$
\begin{aligned}
\overline{I_{n}} & =\int_{0}^{R_{v}} \int_{0}^{2 \pi} I(n, r, \theta) \\
& =\int_{0}^{R_{v}} \int_{0}^{2 \pi} P_{t} r^{\beta \alpha} R_{u}(n, r, \theta)^{-\alpha} \rho_{f r 1} r d r d \theta .
\end{aligned}
$$

Similarly, the uplink ICI variance $\widehat{I_{n}}$ can be obtained by

$$
\widehat{I_{n}}=\int_{0}^{R_{v}} \int_{0}^{2 \pi}\left[P_{t} r^{\beta \alpha} R_{u}(n, r, \theta)^{-\alpha}-\overline{I_{n}}\right]^{2} \rho_{f r 1} r d r d \theta \text {. }
$$

The integrals (7) and (8) can be calculated simply by traditional numerical quadrature tools.

According to (2) and (3), the statistics parameters $\mu_{n}$ and $\sigma_{n}$ for $I_{n}$, and $\mu_{w, n}$ and $\sigma_{w, n}$ for $I_{w, n}$ can be obtained as well. The aggregated ICI $I_{\text {sum }}$ is the sum of independent log-normally distributed variables $I_{w, n}\left(1 \leq n \leq N_{c}\right)$. With commonly used Fenton-Wilkson approximation method, the mean and variance of the normal distribution associated with $I_{\text {sum }}$ can be calculated by

$$
\sigma_{\text {sum }}^{2}=\log \left[1+\frac{\sum_{n=1}^{N_{c}} e^{2 \mu_{w, n}+\sigma_{w, n}^{2}}}{\left(\sum_{n=1}^{N_{c}} e^{\mu_{n}^{2}+\sigma_{w, n}^{2} / 2}\right)^{2}}\right],
$$

$$
\mu_{\text {sum }}=\log \left(\sum_{n=1}^{N_{c}} e^{\mu_{w, n}+\sigma_{w, n}^{2} / 2}\right)-\frac{\sigma_{\text {sum }}^{2}}{2} .
$$

From $\mu_{\text {sum }}^{2}$ and $\sigma_{\text {sum }}^{2}$, the mean and variance of the aggregated ICI $I_{\text {sum }}$ can be obtained by

$$
\begin{aligned}
& \bar{I}_{\text {sum }}=e^{\mu_{\text {sum }}+\sigma_{\text {sum }}^{2} / 2}, \\
& \widehat{I}_{\text {sum }}=\left(e^{\sigma_{n}^{2}}-1\right) \sigma_{\text {sum }}^{2} .
\end{aligned}
$$

\section{B. SINR and spectrum efficiency}

Let $\Upsilon_{f r 1, r}$ denote the SINR of a generic user in the target cell located at distance $r$ away from the target BS for scheme IFR-1, which can be computed by

$$
\Upsilon_{f r 1}(r)=\frac{P_{t} r^{\alpha(\beta-1)} \psi_{0}}{I_{\text {sum }}+\delta^{2}}=\frac{P_{t} r^{\alpha(\beta-1)}}{I_{\text {sum }} / \psi_{0}+\delta^{2} / \psi_{0}},
$$

where $\psi_{0}$ represents the lognormal shadowing between the user and BS 0. It is noted that $\Upsilon_{f r 1, r}$ can be approximated as a lognormal random variable as done with $I_{\text {sum }}$, and its statistics (such as the mean and variance) can be computed accordingly.

Assuming the use of adaptive modulation and coding, we can calculate the average spectrum efficiency (bps/Hz) based on Shannon capacity formula for a user at distance $r$ away from the target BS, which is denoted by $C_{f r 1}(r)$. The spectrum efficiency $C_{f r 1}(r)$ is calculated by

$$
C_{f r 1}(r)=\log _{2}\left[1+\Upsilon_{f r 1}(r)\right] \text {. }
$$

The statistics of spectrum efficiency for scheme IFR-1 can be derived accordingly with (13) and (14). The above equations can be used to calculate the statistics of uplink ICI, SINR and spectrum efficiency for scheme IFR-3, with some minor changes on the number of interfering cells at the $l$ th tier from $N_{f r 1}(l)$ for scheme IFR-1 to $N_{f r 3}(n)$ for scheme IFR-3. The numbers of interfering cells at tiers 1 to 4 are $0,6,6$ and 12 , respectively. Due to the limited space, the equations for scheme IFR-3 are omitted here.

\section{NUMERIC RESULTS}

Simulations were run to verify the analytic model. Typical results with two tiers of interfering cells $\left(N_{c}=18\right)$ are presented. The results with other system configurations showed similar performance trends, and thus they are not presented here. The cells take hexagonal shape in the simulations and have circular shape in the analytic model. Numerical results showed that the approximation used in the analytical model has a negligible impact indeed. Cell size $R_{c}$ is set to $1000 \mathrm{~m}$. Thermal noise density is set to $-174 \mathrm{dBm} / \mathrm{Hz}$. The transmit power for a PRB of $180 \mathrm{kHz}$ is set to $3.55 \mathrm{dBm}$. The standard deviation (measured in $\mathrm{dB}$ ) of the lognormal shadowing is set to $4 \mathrm{~dB}$, which corresponds to $\sigma_{w}=4 / \ln (10)$. In the simulations, the locations of interfering users were generated in a random and uniform way within hexagonal cells.

First, we check the accuracy of the approximation of single cell and aggregated ICI by lognormal random variables. Fig. 2 presents the cumulative distribution function $(\mathrm{CDF})$ of ICI from one cell in the 1st tier and the aggregated ICI for scheme IFR-1, respectively. The presented results were obtained by 
simulations and lognormal distribution fitting with a path loss exponent $\alpha=2.5$ and power compensation factor $\beta=0.5$. It is observed that both single cell and aggregated ICI can be approximated statistically by lognormal distributions very well.

With effective approximation of the ICI by a lognormal distribution, it is expected that our analytical approach can provide a good estimation of the statistics for ICI and the other performance metrics such as SINR. Next, the mean aggregated uplink ICI values for both IFR-1 and IFR-3 schemes are plotted against power compensation factor $\beta$ in Fig. 3. The analytic results match to the simulation results very well. It is observed that with an increase of $\beta$ from 0 to 1 , the uplink ICI increases linearly by about $140 \mathrm{~dB}$ for IFR-1 scheme. Therefore, the power compensation factor has a profound impact on system performance and needs to be configured carefully. Additionally, the path loss exponent $\alpha$ showed its impact on uplink ICI, which is almost identical for IFR-1 and IFR-3 schemes with a fixed $\beta$. Fig. 4 presents the mean SINR results of mobile users located at different distances away from the target BS, for both IFR-1 and IFR-3 schemes. The path loss exponent $\alpha$ is set to 2.5 and 4 . The power compensation factor $\beta$ is 0.5 . Analytical results match very well to the simulation results again. It is revealed that for IFR1 scheme the SINR of mobile users at the edge of a cell is still very low even though power compensation is used. That is due to the fact that while cell edge user signal strength increases with power compensation, the aggregated interference also increases quickly. Therefore, power compensation alone could not solve the cell edge user QoS problems, and effective frequency reuse schemes are required. We believe our analytic model can serve as an effective tool for evaluation and optimization of these schemes.

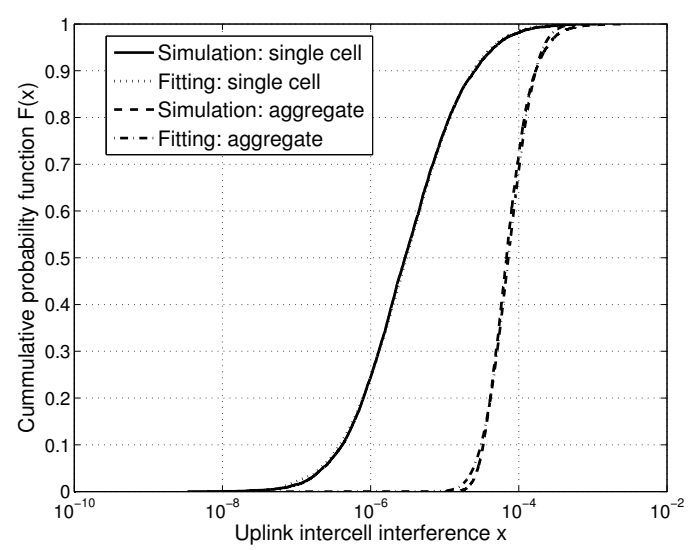

Fig. 2. Cumulative probability function (CDF) of a single cell and aggregated uplink ICI for IFR-1 scheme, with a path loss exponent $\alpha=2.5$ and power compensation factor $\beta=0.5$.

\section{ACKNOWLEDGEMENTS}

The work was supported partially by the UK Engineering and Physical Sciences Research Council (EPSRC) under grant EP/1010157/1, the National Natural Science Foundation of China (NSFC) under grant 61103177, and Taiwan National Science Council research grant NSC99-2221-E-006-016-MY3.

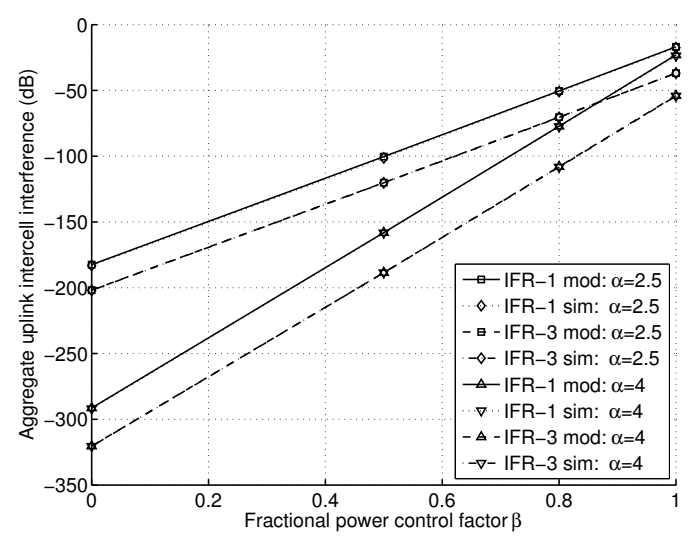

Fig. 3. Mean aggregated uplink ICI $(\mathrm{dB})$ versus power compensation factor $\beta$ in both IFR-1 and IFR-3 schemes.

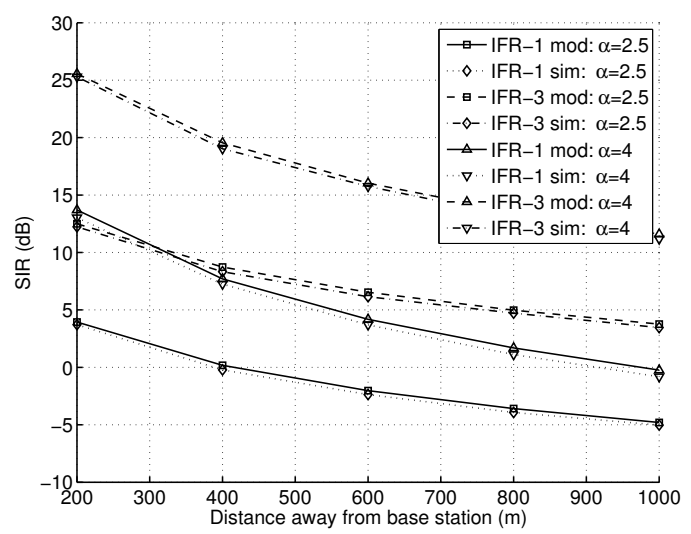

Fig. 4. Mean SINR (dB) versus user distance away from a target base station for IFR-1 and IFR-3 schemes, with $\beta=0.5$.

\section{REFERENCES}

[1] H. Holma and A. Toskala, "LTE for UMTS: OFDMA and SC-FDMA based radio access," John Wiley \& Sons Ltd, 2009.

[2] T. Novlan, R. Ganti, A. Ghosh, and J. Andrews, "Analytical evaluation of fractional frequency reuse for OFDMA cellular networks," IEEE Transactions on Wireless Communications, Vol. 10, No. 12, pp. 42944305, Dec. 2011

[3] T. Bonald and A. Proutiere, "Wireless Downlink Data Channels: User Performance and Cell Dimensioning," In Proc. Mobicom'03, 2003.

[4] J. Kelif, M. Coupechoux, and P. Godlewski, "On the Dimensioning of Cellular OFDMA Networks," Physical Communication, Vol. 5, No. 1, pp. 10-21, 2012.

[5] J. Andrews, F. Baccelli, and R. Ganti, "A tractable approach to coverage and rate in cellular networks," IEEE Transactions on Communications, Vol. 59, No. 11, pp. 3122-3134, Nov. 2011.

[6] A. Wyner, "Shannon-theoretic approach to a Gaussian cellular multiaccess channel," IEEE Transactions on Information Theory, Vol. 40, No. 6, pp.1713-1727, Nov. 1994.

[7] J. Xu, J. Zhang, and J. Andrews, "On the accuracy of the Wyner model in downlink cellular networks," In Proc. IEEE ICC'11, 2011.

[8] S. Elayoubi and O. Haddada, "Uplink Intercell Interference and Capacity in 3G LTE systems," In Proc. IEEE ICON'07, 2007.

[9] M. Karray, "Evaluation of the blocking probability and the throughput in the uplink of wireless cellular networks," In Proc. IEEE ComNet'10, 2010.

[10] M. Coupechoux and J. Kelif, "How to set the fractional power control compensation factor in LTE?" in Proc. of IEEE Sarnoff Symposium, May 2011.

[11] H. Tabassum et al, "A Framework for Uplink Intercell Interference Modeling with Channel-Based Scheduling," IEEE Transactions on Wireless Communications, Vol. 12, No. 1, pp. 206-219, Jan. 2013.

[12] C. Castellanos et al, "Performance of uplink fractional power control in UTRAN LTE,' In Proc. of IEEE VTC'08, 2008. 